BULLETIN Bulletin hispanique

HISPANIQUE Université Michel de Montaigne Bordeaux

$115-2$ | 2013

Les traductions vieillissent-elles?

\title{
«La literatura en català millora traduïda»
}

Realitat i mite d'un tòpic

\section{Giuseppe Grilli}

\section{(2) OpenEdition}

Journals

Electronic version

URL: http://journals.openedition.org/bulletinhispanique/2766

DOI: 10.4000/bulletinhispanique.2766

ISSN: 1775-3821

Publisher

Presses universitaires de Bordeaux

\section{Printed version}

Date of publication: 28 December 2013

Number of pages: $589-598$

ISBN: 978-2-86781-908-7

ISSN: 0007-4640

Electronic reference

Giuseppe Grilli, « «La literatura en català millora traduïda» », Bulletin hispanique [Online], 115-2 | 2013,

Online since 14 February 2017, connection on 19 April 2019. URL : http://journals.openedition.org/

bulletinhispanique/2766 ; DOI : 10.4000/bulletinhispanique.2766 


\title{
«La literatura en català millora traduïda». Realitat i mite d'un tòpic
}

\author{
Giuseppe Grilli \\ Università degli Studi Roma Tre
}

Dans ce travail on étudie différentes traductions de poésies de quelques auteurs catalans $d u X X$ siècle en italien. Il s'agit de traductions faites par des poètes ou des écrivains fameux, par exemple Montale pour Maragall, ou bien par des traducteurs connus, comme pour Riba et Rodoreda. Le dernier texte est une prose de J. F. Mira.

Mots-clés : Traduction, Maragall, Riba, Rodoreda, Mira.

En nuestro trabajo estudiamos diversas traducciones poéticas de autores catalanes del siglo XX al italiano. Son traducciones realizadas por poetas y escritores de renombre, por ejemplo en el caso de Montale a propósito de Maragall, o de traductores experimentados y bien conocidos, como ocurre en el caso de Riba, o Rodoreda. El último texto es una prosa de J.F. Mira.

Palabras clave: Traducción, Maragall, Riba, Rodoreda, Mira.

This paper analyses different translations of 20th Century poems, by Catalan authors, into Italian. The translators are either very important poets like Montale, or professionals in translations, as in Riba's and Rodoreda's poems. The last text is a prose one from J.F. Mira.

Keywords: Translation, Maragall, Riba, Rodoreda, Mira.

$E^{\prime}$

1 tema de les traduccions, com és massa sabut, es desenvolupa segons una problemàtica escorredissa: ja d'entrada se'ns fa difícil esbrinar on col-locarlo, cada cop que ens situem en terrenys pròxims però separats, con la lingüística, la literatura o la ara recent traductòlogia. No entraré en els aspectes teòrics que en els últims anys han experimentat uns importants avenços i que, de fet, 
han modificat els nostres hàbits respectes a la qualitat de les traduccions i a les seves finalitats. Tanmateix em permeto recordar aquella cèlebre i inoblidable censura cervantina respecte a les traduccions "facils», és a dir aquelles que impliquen el passatge d'una llengua a l'altra dins d'una mateixa família -que és el cas de les llengües romàniques-. En efecte en textos originals en català i l'italià, especialment si ens referim a textos literaris de qualitat i els poètics, en particular, podríem argumentar que la traducció s’apropa més al comentari que a la translació de formes i significats. El lector culte, que és el lector habitual de la poesia, o de la prosa d'una certa implicació complexa, de fet no té grans dificultats en entendre un text d'una o altra llengua: un català pot llegir un text italià sense gaires entrebancs i viceversa a un italià no se li presenta misteriós un full escrit en català. D'altra banda, els llibres escrits en català, per raons que ara i ací es poden al-ludir només de passada, tenen un públic seleccionat, un públic que ha elegit abans de llegir. Vull dir que el lector que s'apropa a la literatura catalana normalment sap que no hi trobarà el best seller trivial que ocupa majoritàriament la producció i la venta dels objectes de l'entreteniment contemporani. De fet els llibres, concretament els poètics o també els que no ho son explícitament, tenen el seu mercat en edicions bilingües. En aquestes la traducció, a més de ser un suport econòmic en el sentit d'estalviar al lector la recerca d'un mot o d'una locució (unitat fraseològica o parèmia) de percepció no immediata, funciona com a lectura guiada, com a primera aproximació interpretativa.

Si aquesta hipòtesi o premissa inicial té validesa, podem de seguida afirmar, ja des d'ara, sense entrar en detalls, que les traduccions del català sí que envelleixen, $\mathrm{i}$ han envellit molt, donat el tarannà d'una literatura que ha vist la seva continuïtat històrica moderna sotragada per esdeveniments tan radicals. I que, en concret, sols des de fa unes poques dècades comença a acumular-se un gruix de consciència literària, o sigui d'una estable formació crítica i d'una hermenèutica consolidades. Amb això, podem afirmar que la interpretació de textos importants de la literatura moderna, així com de la clàssica (o neoclàssica), ha anat modificant-se notablement amb el creixement esplendorós d'una investigació que ha gaudit, com mai abans, de tesi, congressos i simposis, estudis i revistes.

Paral-lelament a aquests aspectes significatius, que impliquen profundament ús i recepció de les traduccions dels textos literaris catalans, crec que mereix atenció un fet en certa mida paradoxal: hem dit que la literatura catalana que aconsegueix, o que pot aspirar a tenir un mercat, encara que d'àmbit restringit, és una literatura de gran qualitat. Però es tracta, òbviament, d'una literatura que ambiciona un públic que va més enllà de l'encara més reduït nombre de lectors que dominen la llengua original i que, per tant, no necessiten cap mena de versió i de perifrasis o, almenys, d'un comentari.

Ara, donat tots aquests aspectes que he resumit molt ràpidament, hom ha cregut intervenir sobre els textos catalans amb adaptacions i formes de l'expressió que els fessin de volta en volta més atractius, intrigants, o més símils o digeribles per un públic que justament, perquè pertany a un veïnat 
immediat, cerca, o es creu que cerca, una motivació suplementària. Podem dir que es tracta de necessitats que han motivat els traductors i fins i tot els lectors. Malgrat s'hagi argumentat que la caracteristicitat, o sigi la especifica essència mateixa -la poesia catalana com a reducte clos i autoprotegit- és l'autèntica força de la llengua, com recorda Montale tot comentant el seu assaig de traducció del Cant Espiritual de Maragall, de fet ell és el primer en adaptar el text a una homologació d'abast europeïtzant ${ }^{1}$. Cercaré de fer entendre el tema amb uns pocs exemples que espero significatius. Però abans d'aquest modest assaig analític, em permeto introduir una anècdota molt recent que he recollit tan sols fa uns dies al magne Congrés dedicat al primer centenari de la mort de Joan Maragall. En la seva intervenció Narcís Comadira recollia i feia seva la censura envers l'ús descurat i massa influït pel parlar barceloní de tanta part de la producció lírica maragalliana. I es va aprofitar com exemple que be s'escau a la necessitat de polir un text a vegades de escassa intel.ligibilitat o de dubtosa eficàcia lingüística de la supressió d'un vers a la traducció d'Eugenio Montale del Cant espiritual:

Si per mi com aquest no n’hi haurà cap!

Un vers que es mereixia d'anar a l'infern, en opinió de Comadira seguint o interpretant a Montale, al fi de salvar un poema que, per la seva força, segurament valia la pena traduir ${ }^{2}$. I aquí afegiria jo que l'acte de traducció no sols implicava el trasllat a una altra llengua, sinó decididament a l'adscripció del poema -i del poeta- un sistema homologable a la koiné de la poesia europea del Nou-cents.

Donat que ja he citat a Montale, vull recordar que les pàgines que va escriure sobre la cultura catalana arran d'un viatge fet com a enviat periodista (d'una mena que avui podem dir extingida) comenta que la altíssima tradició lírica moderna veu personalitats de valor encara arrelades a una escriptura ex abundantia cordis, com Maragall o Carner, i altres, essencialment Carles Riba, autènticament en sintonia amb el nou verb de la poesia més pura. És a dir (interpreto) un poeta que ha escoltat o escolta la lectio de Valery ${ }^{3}$. Bé, els

1. «A lavoro finito si vede (...) che è andato perduto il meglio, quel suono scoppiettante di pigna verde buttata nel fuoco che è proprio di tutta la poesia catalana " (Il Mondo Europeo, III, 39, 1947, p. 15. Cal dir que aquesta actitud -sense que Montale en tingui una idea sinó molt vaga- era a la base del desprestigi del català literari a les polèmiques del segle XVI; també cal dir la brevetat i duresa foren -alhora, per paradoxa, també- arguments a favor.

2. La nova traducció de Lello Voce, que obre l'edició italiana recent dels Elogis de la paraula $i$ de la poesia el reincorpora, en això restaurant el criteri seguit per Wilcok a la seva antologia Poeti catalani: «Se per me, come questo, mai ce ne sarà! ».

3. Vaig avançar un comentari a un ja antic article meu: «Montale, Maragall i la via catalana a la poesia», Els Marges, $n$. 8 (set. 1975, pp. 107-118). Aquest breu treball va tenir una eco important al llibre de Loreto Busquets que el va increpar críticament amb remarques de tota mena, que tanmateix -amb la distància dels anys- no em sembla que la estudiosa argumenti cap alternativa substancial respecte a la meva interpretació de la versió montaliana. Només un comentari: Busquets jutja fora de lloc el fet que senyalés el pas del Vos al tu en la traducció no 
traductors de Riba a l'italià s'ho han pres a la lletra. I cal dir que ho han fet bé, o ho ha fet bé, si ens referim -com de fet em refereixo- a Giuseppe E. Sansone, autor d'una versió bilingüe -per la prestigiosa col-lecció de poesia Einaudil'any $1977^{4}$ de la cèlebre i emblemàtica Elegia IX, justament la que ve dedicada a Pompeu Fabra i que recull el mite de la llegua poètica com a pàtria; es tracta del concepte que es sintetitza al dístic final:

Sols que té menys espera i arrenca de tots els exilis cap al seu crit, i els batuts van retrobant-se soldats

Ma pazienta di meno e da tutti gli esili il suo grido ci trappa: chi fu vinto si trova di nuovo guerriero (Sansone)

Si comparem aquesta bella versió de Sansone amb la que va realitzar Costrafreda al castellà, veurem com aquesta darrera respon molt més a un criteri (no sols a una literalitat) propi del text original:

sólo que espera menos y arranca desde todos los exilios

hacia su grito, y los vencidos van reencontrándose

(Costafreda)

Tanmateix, voldria destacar qualque altre exemple simptomàtic d'una actitud on traduir és dur el text originari dins del sistema de la recepció, i tal volta l'operació es justifica per part de les actituds del propi autor ${ }^{5}$.

té significació ja que al Pater noster ja es manifesta el mateix. Vol dir que Montale sabia o va investigar el tractament amb Déu en català a la liturgia?

4. Un comentari relatiu a la elegia es troba al meu 'assaig' presentat a la I Giornata di Studi Ispanici del Mediterraneo (en premsa).

5. No és un cas que poetes come Riba (i més tard Comadira) hagin volgut introduir poemas escrits per exemple, directament en italià. Com a completament en faig aquí una mostra encarada:

Non destar le speranze fuggitiva dormenti in grembo a una infrangibil fede: ogni antico spavento ormai lor cede, nel muto sonno eternamente vive.
Sera

O assurdo amore mio,

La tua mente veloce

Distrugge questo atroce Inganno ch'è il desio.

La luce, poi, ci manca;

E il cuore ci si stanca.

Em sembla evident, el que vol dir intencional i deliberat, el ressò leopardià al text de Riba, i el de Pavese (passat per Ungaretti, en estranya combinació gairebé ossimòrica) al de Comadira. 
Passo a considerar dos exemples, el primer és el de l'Elegia cinquena:

Clou-te, cúpula verda per sobre el meu cap cristal.lina!

Aigües de curs discret, brisa que a penes ets més

que un moviment del silenci, imiteu la manera senzilla

com la meva sang ara s'oblida i jo sé.

L'inacabable somni del mon endolceix una a una

ses onades entorn del malencònic jardí.

Dins la meva ànima en pau sóc el nàufrag que en l'illa profunda

on reneix de la mar, súbitament reconeix

una pàtria d'antany; i no en té sorpresa; el crepuscle

fa més pur el sender -oh pueril! Oh reial!

que l'ha tornat a prendre, envellit i nu, però en flama

més a cada pas, més alentint cada pas

perquè vol la nit, i arribar a l'esposa secreta

com esperat d'un esclat sempre imminent de l'enyor,

i ésser l'un per l'altre un do amorós del misteri

- nit amb joia dels ulls, nit més enllà de la nit!

C. Riba
Chiuditi, cupola verde sul mio capo cristallina!

Acque dal corso discreto, brezza che appena sei piú

d'un moto del silenzio, imitate la semplice maniera

come ora il mio sangue s'oblia e io so.

Il sogno infinito del mondo fa dolce una a una

le sue onde tutt'intorno al triste giardino.

Nella pace del cuore naufrago sono che nell'isola profonda

dove il mare rinasce, all'istante ravvisa una patria remota; e non si stupisce; l'imbrunire

fa piú puro il sentiero -oh puerile! oh reale!

che ha ripreso, nudo e piú vecchio, fiammeggiante però

ogni passo di piú e sempre di piú rallentando

perché vuole la notte, e arrivare alla sposa segreta

come voluto da scoppio di nostalgia ognora imminente,

e l'uno per l'altro del mistero esser dono amoroso

-notte con la gioia negli occhi, notte ancor oltre la notte!

Però aquests mecanismes que acabem de comentar es manifesten encara més acusats en el cas de l'Elegia IV, una de les primeres i plenament involucrada en el clima dels primers moments de l'exili. Recordo de passada el fet, prou conegut, que l'Elegia IX es correspon ja al clima del retorn a Catalunya. Vegeu: 


\section{ELEGIA IV}

Pura en la solitud i en l'hora lenta, una dona

fa lliscà, amb moviment d'arbre o de crit amorós,

al llarg dolç dels braços alçats, la túnica.

Mentre

brilla ja el tors secret, resta captiva en el lli, dalt, la testa. Un instant o dos. Ah! ¿són prou perquè es trenqui

foscament el lligam entre la bella i aquest tímid juny que d'ella, nua dins l'ona, esperava joia i impuls fluvial per a perfer-se? ¿Han estat prou, que tu, imponderable cosa d'or i mirada,

testa, flor dreta, en surts vaga —i talment reguardant,

ara, els no-res del silenci que eren adés

venturosos

còmplices? Un cucut canta de sobte, innocent.

Ella somriu. La sang juvenil del món torna a

córrer,

salta, brusca, amb el salt de la magnífica, i va

temps avall, cap a sols més madurs — i ella

neda, oh ritme!

cap a l'estiu excessiu - ella i els déus i els meus ulls!

[1939]

\section{ELEGIA IV}

Pura nella solitudine e nell'ora lenta, una donna

fa scivolare, con moto di albero o di grido amoroso,

dolce, lungo le braccia innalzate, la tunica. Mentre

già brilla il busto segreto, in alto, prigioniera del lino

rimane la testa. Un attimo o due. Ah!

Basta per rompere

foscamente il legame fra la bella e questo

timido giugno che da lei attendeva, nuda nell'onda,

gioia ed impulso fluviale per farsi perfetto? È bastato

dato che tu, imponderabile cosa di oro e di sguardo,

testa, fiore dritto, ne sorgi indecisa come temendo

il nulla del silenzio ora, complice fausto di prima?

Un cuculo canta d'improvviso, innocente.

Lei sorride. Torna a scorrere il sangue giovane del mondo,

salta, brusco, come la magnifica, e corre avanti nel tempo

verso soli più maturi - e lei nuota, oh ritmo!

verso l'estate eccessiva - lei e i miei occhi e gli dèi!

Un cas prou different el trobem en un text de Pere Gimferrer. Es fa evident -més enllà del giny del traductor o de la seva imperícia- que la versió italiana apareix com a simplificadora, banalitzada respecte a l'original que mostra una riquesa i una dispersió de recursos que tal volta justament ha induït a formular l'etiqueta de poètica veneciana: 


\begin{tabular}{|c|c|}
\hline $\begin{array}{l}\text { LISTEMES } \\
\text { La poesia és } \\
\text { un sistema de miralls } \\
\text { giratoris, lliscant amb harmonia, } \\
\text { desplaçant llums i ombres a l'emprovador: per } \\
\text { què } \\
\text { el vidre esmerilat? } \\
\text { Com parlant - de conversa } \\
\text { amb les tovalles i música suau - jo et diria, } \\
\text { estimada, } \\
\text { que aquest reflex, o l'altre, és el poema, } \\
\text { o n'és un dels aspectes: hi ha un poema } \\
\text { possible } \\
\text { sobre la duquessa morta a Ekaterinenburg, } \\
\text { i quan es mou el sol vermell a les finestres, jo } \\
\text { recordo } \\
\text { els seus ulls blaus ... No ho sé n'he passat } \\
\text { tantes, d'hores, } \\
\text { als trens de nit, tot llegint novel-les policíaques } \\
\text { (sols a la casa buida, obríem els armaris), } \\
\text { i una nit, anant cap a Berna, dos homes es } \\
\text { besaren al meu departament } \\
\text { perquè era buit, o jo dormia, o era fosc } \\
\text { (una mà cerca l'altra, un cos l'altre) } \\
\text { i amaga aquest aspecte: el real i el fictici, } \\
\text { la convenció, és a dir, i les coses viscudes, } \\
\text { l'experiència de la llum als boscos hivernals, } \\
\text { la dificultat de posar coherència -és un joc de } \\
\text { miralls-, } \\
\text { els actes que es dissolen en la irrealitat, } \\
\text { els àcids que envaeixen velles fotografies, } \\
\text { el groc, la lepra, el rovell i la molsa que } \\
\text { esborren les imatges, } \\
\text { el quitrà que empastifa les cares del nois amb } \\
\text { canotier, } \\
\text { tot allò que una tarda morí amb les bicicletes, } \\
\text { cromats vermells colgats a les cisternes, } \\
\text { a càmara lenta els cossos (a l'espai, com al } \\
\text { temps) sota les aigües. } \\
\text { (Enfosquit com el fons d'un mirall esberlat, } \\
\text { l'emprovador } \\
\text { és l'eix d'aquest poema.) } \\
\text { Obra Catalana Completa. Poesia. Barcelona: } \\
\text { Edicions 62, 1995: } 110 .\end{array}$ & $\begin{array}{l}\text { LISTEMI } \\
\text { La poesia è } \\
\text { un sistema di specchi } \\
\text { rotatori, che scivolano armonici, } \\
\text { che spostano luci ed ombre nel salottino di } \\
\text { prova: perché } \\
\text { il vetro smerigliato? Come parlando } \\
\text { conversando } \\
\text { con la tovaglia e musica soave - ti direi, mia } \\
\text { cara, } \\
\text { che questo riflesso, o l'altro, è la poesia } \\
\text { o ne è uno degli aspetti: c'è una poesia } \\
\text { possibile } \\
\text { sulla duchessa morta a Ekaterinenburg, } \\
\text { e quando si muove il sole rosso nelle finestre, } \\
\text { io ricordo } \\
\text { i suoi occhi azzurri... Non so, ne ho passate } \\
\text { tante, d'ore, } \\
\text { nei treni di notte, leggendo romanzi } \\
\text { polizieschi } \\
\text { (soli nella buia casa, aprivamo gli armadi), } \\
\text { e una notte, andando a Berna, due uomini si } \\
\text { baciarono nel mio scompartimento } \\
\text { perché era vuoto, o io dormivo, o era buio } \\
\text { (una mano cerca l'altra, un corpo l'altro) e } \\
\text { adesso gira il cristallo } \\
\text { e nasconde questo aspetto: di realtà e di } \\
\text { finzione, } \\
\text { la convenzione, vale a dire, e le cose } \\
\text { vissute, } \\
\text { l'esperienza della luce nei boschi invernali, } \\
\text { la difficoltà di dare coerenza -è un gioco di } \\
\text { specchi-, } \\
\text { gli atti che si dissolvono nell'irrealtà, } \\
\text { gli acidi che invadono vecchie fotografie, } \\
\text { il giallo, la lebbra, la ruggine e il muschio } \\
\text { che cancellano le immagini, } \\
\text { il catrame che imbratta le facce dei ragazzi } \\
\text { in canotier, } \\
\text { tutto ciò che un pomeriggio morì con le } \\
\text { biciclette, } \\
\text { rossi cromati sepolti in cisterne, } \\
\text { a camera lenta i corpi (nello spazio, come } \\
\text { nel tempo) sotto le acque. } \\
\text { (Offuscato come il fondo d'uno specchio } \\
\text { infranto, il salottino } \\
\text { è l'asse di questa poesia.) } \\
\text { Antologia della poesia spagnola (castigliana, } \\
\text { catalana, galega, basca) (dal } 1961 \text { ad oggi), a } \\
\text { cura di Rosa Rossi e Valentí Gómez i Oliver,, } \\
\text { Nuove Amadeus Edizioni, Cittadella, } \\
1996 .\end{array}$ \\
\hline
\end{tabular}


Un cas que podríem col-locar com al mig d'aquest itinerari exemplificatiu l'ofereix un sonet de Mercè Rodoreda pertanyent a la sèrie o llibre inèdit El món d'Ulises, malgrat que els materials que podia encabir son ara públics i publicats. En aquest cas la traducció (d'Anna Maria Saludes, a punt de sortir, però ara i adés inèdita) és significativa del caràcter d'assaig que té l'obra poètica original. No hi ha dubte que aquesta -com la gran part de la producció literària catalana escrita a l'exili- fou ideada en condicions de llibertat limitada, i l'autora era impel.lida per exigències vitals prou acusades d'indigència $\mathrm{i}$ incomoditat: el poemes es feien sota la suggestió d'una festa i d'un premi, migrat però essencial per equilibrar el pressupost d'una parella (Obiols i Rodoreda) que, com altres de l'exili, passaven moments d'angoixa de tota mena. L'estil que en deriva és alhora treballat i provisional, i s'ofereix al traductor com un terreny òptim d'interpretació i execució. Vet aquí el resultat:

\section{[ Ciclop]}

Rodava dintre l'ull, oh ma indefensa ovella,

l'estella olivera trempada al foc del pi;

l'illa que has trepitjat t'ha dat, reial mastí

més dolça que el teu vi la meva sang vermella.

Passaràs tu, Ningú, tot el dolor t'espera, cremant l'òrbita exalta el vent blau del llorer,

les meves mans són cegues i no poden desfer

el grop del pensament, petit cadell de fera.

Esguard buidat de verd i d'alts crepuscles rosa,

una pau vegetal dintre la boca em posa,

silenci de colors, udol desmesurat...

Venja'm, rocall del cim; i tu, pacient entranya,

demana als déus que et reblin al fons del seu passat

illes d'enyor que facin la seva terra entranya.

\section{[ Ciclope]}

Ruotava nell'occhio, mia dolce pecorella inerme,

La sgargiante stella d'olivo molata col fuoco d'un pino;

Lisola che hai calpestato t'ha offerto, o mastino regale,

Più del vino dolce il mio sangue vermiglio.

Supererai, Nessuno, tutto il dolore t'attende,

Bruciando l'orbita esalta il vento azzurro dell'alloro,

Le mani sono cieche per me e non potranno disfare

Il nodo del pensier mio, mio piccolo cucciolo di fiera.

Lo sguardo svuotato di verde e di alti crepuscoli rosa,

Una pace vegetale si adagia sulla mia bocca.

Silenzio dei colori, urla smisurate...

Rocce in su la cima, a voi chiedo vendetta; o viscere pazienti

Chiedete agli dei che ti accolgano nel pozzo del loro passato

Isole di nostalgia che rendano straniera la patria.

Finalment voldria fer un exemple de prosa. Es tracta del incipit de la novel.la de J. F. Mira que gira a l'entorn de Roderic Borja el cèlebre papa Alexandre VI, 
Borja Papa. La traducció, publicada, és de Nancy De Benedetto. Hi trobem un difícil canvi d'estil i de subjecte gramatical. Provem a descriure el pas:

El vint-i-sis d'agost de l'any del Senyor mil quatre-cents noranta-dos em van coronar papa, era un dia de molta calor, com si el cel hagués volgut també en això afegir-me a la llista dels quatre successors del meu oncle Calitx, que tots van ser coronats en un d'aquests dies de gran calda de Roma, volia dir que cinc pontífexs seguits havien deixat la vida i el regne per no haver resistit un estiu més de calor enganxosa i de mal aire, qui sap si jo seré el sisè que tampoc no arribarà a un altre setembre, a mig matí vingué el cubiculari a despertar-me, Santedat, és l'hora, Santedat, i em va trobar en camisa i sense tapar amb el llençol, però un papa en camisa és un papa, Santedat, feia quinze dies que em deien Vostra Beatitud, Santíssim Pare, tots excepte el meu cosí Francesc. Sa Santedat s'hauria estimat més ser coronat un dia d'aire clar d'hivern, o millor encara un mati de primavera tèbia, però no es pot triar el millor temps de l'any per accedir al papat, i més valia gran calor que gran fred, pensant en el poble de Roma i en la llarga cavalcada, i millor sol que no pluja.
Il ventisei agosto dell'anno del Signore mille quattrocento novantadue fui incoronato papa, era un giorno di intenso calore, come se il cielo avesse voluto anche in questo aggiungermi alla lista dei quattro successori di mio zio Callisto, che erano stati tutti incoronati in una di queste giornate romane di gran caldo, il che significava che cinque pontefici di seguito avevano lasciato la vita e il regno per non aver resistito a un'ennesima estate di caldo afoso e malsano, chi può dire se non sarò io il sesto a non arrivare a un altro settembre, intorno alle dieci venne a svegliarmi il cubicolario, Santità è l'ora, Santità, mi trovò in camicia e senza lenzuolo addosso, ma anche in camicia un papa è un papa, Santità, da quindici giorni mi chiamavano Vostra Beatitudine, Santissimo Padre, tutti tranne mio cugino Francesco. Sua Santità avrebbe preferito essere incoronato in un chiaro giorno d'inverno o, meglio ancora, una mattina tiepida di primavera, ma non è possibile scegliere il tempo migliore dell' anno per diventare papa, meglio il gran caldo poi che il gran freddo, pensando al popolo di Roma e alla lunga sfilata, meglio il sole che la pioggia.

Com hem pogut observar, si ens fixem en el text original i la traducció, que proposo encarats, la dispositio dels mots traduïts tanmateix amb total fidelitat que quasi ens podria fer pensar en una voluntat de calc, d'alguna manera en canvi 'estilitza' l'original, tot simplificant uns trets que anomenaríem -vulgarment- barrocs, mimètics tal volta de la 'valenciana prosa', doncs, d'un efecte pràcticament intraduüble en italià, al no fer malabarismes amb els llenguatges regionals del segle $\mathrm{XV}$, incomprensibles per un públic modern $\mathrm{i}$ general. Ho veiem aixì a l'exemple: "in un chiaro giorno d'inverno o, meglio ancora, una mattina tiepida di primavera ", frase a la qual corresponia en català el gir, un xic més complexe i colorit: " un dia d'aire clar d'hivern, o millor encara un mati de primavera tèbia ", on juga un rol significatiu la organització de les paraules, més encara que la selecció de les mateixes. Si la poesia és un sistema de miralls, segon la definició suggerent de Pere Gimferrer, traduir poesia és fer girar els miralls, com efectivament ho feien en les seves construccions metamètriques, tan volgudes per Caramuel, els seus deixebles catalans, prou enginyosos que jo mateix he citat a la Antologia dels Poetes Catalans, i obtenir projeccions de objectes tridimensionals. 


\section{Bibliografia}

Per donar unes indicacions metodològiques i fer explicites les al-lusions intercalades en el text, recullo a continuació la bibliografia de referència en la qual he tractat temes vinculats amb els aspectes temàtics que son objecte dels arguments exposats aquí.

Antologia dels Poetes Catalans, dirigida per M. de Riquer, vol. II a cura de Grilli Giuseppe, Del segle XVI a Verdaguer, Barcelona, Galaxia Gutenberg, 1998.

Grilli Giuseppe, «Montale, Maragall i la via catalana a la poesia», Els Marges, 8, 1976, pp. 109-113.

— «La querelle sul catalano lingua letteraria», L’Albero, 59, 1978, pp. 189-193.

— «Poetica e lingua comune in Catalogna da C. Riba ad oggi», dins XIV Congresso Internazionale di Linguistica e Filologia Romanza, Atti, vol. V, Napoli, Macchiaroli, 1981, pp. 99-111.

— «Gabriel Ferrater lettore di Carles Riba», AION-SR, XXVII, 1985, pp. 117-129.

— «Poesia artificiosa e metametrica nella letteratura catalana», AION-SR, XXVII, 1985, pp. 93-355.

— «De Gabriel Ferrater a Joan Maragall», in Oller D. -Subirana J.(eds.), Gabriel Ferrater "in memoriam”, Barcelona, Proa, 2001, pp. 199-206.

— «Traducciones de la Comedia : un desafío catalán», dins Muñiz Muñiz M. de las Nieves (ed.), La traduzione della letteratura italiana in Spagna (1300-1939), Atti del Primo Convegno Internazionale, Firenze, F. Casati, 2008, pp. 543-552.

- «El que és menut és maco: una reflexió sobre la interpolació narrativa 'minimal' en el textos maragallians", dins Terricabras Josep-Maria (ed.), Joan Maragall. Paraula i pensament, Girona, Editorial Documenta Universitària, 2011, pp. 123-138.

— "Gandia, els Borgia, Ausias March», dins De Benedetto Nancy e Ravasini Ines (ed), Da Papa Borgia a "Borgia papa". Letteratura, lingua e traduzione a Valencia, Lecce, Pensa, 2010, pp. 27-42.

— "Carles Riba: humanista i viatger», dins Miralles Carles, Malé Jordi, Pujol Pardell Jordi (ed), Actes del III Simposi Carles Riba (Barcelona, 30 de novembre i 1 i 2 de desembre de 2009), Barcelona, Institut d'Estudis Catalans, 2012, pp. 31-48. 